Research Article

\title{
Identifying PIF1 as a Potential Target of Wenxia Changfu Formula in Promoting Lung Cancer Cell Apoptosis: Bioinformatics Analysis and Biological Evidence
}

\author{
Xiangjun Yin, ${ }^{1}$ Dongfang Kan, ${ }^{2}$ Jiazhao Ruan,, Delong Wang, ${ }^{1}$ Yi Chai, ${ }^{1}$ Shengqi Huang, \\ Beiying Zhang, ${ }^{1}$ Jixin Wang, ${ }^{3}$ and Xuming Ji $\mathbb{D}^{1,4,5}$ \\ ${ }^{1}$ School of Basic Medical Sciences, Zhejiang Chinese Medical University, Hangzhou 310053, China \\ ${ }^{2}$ Innovative Institute of Chinese Medicine and Pharmacy, Shandong University of Traditional Chinese Medicine, Jinan 250355, \\ China \\ ${ }^{3}$ Zhejiang University-University of Edinburgh Institute, Zhejiang University, Hangzhou 310058, China \\ ${ }^{4}$ Key Laboratory of Neuropharmacology and Translational Medicine of Zhejiang Province, Hangzhou 310053, China \\ ${ }^{5}$ Academy of Chinese Medical Sciences, Zhejiang Chinese Medical University, Hangzhou 310053, China
}

Correspondence should be addressed to Xuming Ji; jixuming724@163.com

Received 24 March 2021; Accepted 8 September 2021; Published 25 September 2021

Academic Editor: Shao-Hsuan Kao

Copyright (C) 2021 Xiangjun Yin et al. This is an open access article distributed under the Creative Commons Attribution License, which permits unrestricted use, distribution, and reproduction in any medium, provided the original work is properly cited.

\begin{abstract}
Lung cancer remains the leading cause of cancer-related deaths worldwide. Traditional Chinese medicine (TCM) is a valuable resource of active natural products and plays an important role in cancer treatment with the advantages of high efficiency and safety. Wenxia Changfu formula (WCF) is modified from Dahuang Fuzi decoction from Han Dynasty and has been used for treating lung cancer in China. Our previous research showed that WCF had an antitumor effect in vivo and in vitro, while the mechanism has not been well illustrated. In this study, the effect of WCF on the proliferative ability in three lung cancer cells and one noncancerous human cell line was evaluated by 3-(4,5-dimethylthiazol-2-yl)-2,5-diphenyltetrazolium bromide (MTT) assay. WCF suppressed A549, H460, and PC-9 cell viability in a dose-dependent manner, with no inhibition of noncancerous MRC-5 cells after $48 \mathrm{~h}$ treatment with WCF $(0-50 \mathrm{mg} / \mathrm{mL})$. Furthermore, we screened for genes in A549 cells using four WCF-treated samples and four control samples on a gene expression profile microarray. 21 genes were significantly downregulated by WCF, which may potentially play an important role in the proliferation of A549 cells. High-content screening evaluated whether silencing the 21 genes affected A549 cell growth. The results showed that PIF1 knockdown exhibited the most potent inhibition of cell proliferation compared with the other genes. Downregulation of PIF1 increased A549 cell apoptosis and the activity of caspase 3/7. Besides, RT-PCR showed that the expression levels of PIF1 mRNA decreased significantly in A549, H460, and PC-9 cells after WCF treatment. In conclusion, the present observations indicate that WCF may inhibit lung cancer cell proliferation by promoting apoptosis via regulating the expression of PIF1.
\end{abstract}

\section{Introduction}

Lung cancer remains one of the most frequently diagnosed cancers and is the leading cause of cancer-related deaths, with $>1.7$ million deaths worldwide $[1,2]$. Among these cases, $80-85 \%$ of the total incidence is non-small-cell lung cancer (NSCLC) [3, 4]. Over $60 \%$ of lung cancer patients are diagnosed at an advanced stage when the tumor cannot be treated by surgical resection any more, chemotherapy and radiation therapy are still the mainstays of treatment for patients with lung cancer $[5,6]$. Despite recent advances in the diagnosis and treatment of lung cancer, the five-year survival rate remains $\sim 23 \%$ [7]. It is still urgent to discover potential therapeutic strategies to improve the prognosis of lung cancer patients [8].

Natural products have played a key role in drug discovery of cancer [9]. It was found that $62(33.5 \%)$ of the total number of small-molecule anticancer drugs approved by 
FDA in the past 39 years were derived from natural products or their derivatives [10]. Compared with typical synthetic small-molecular libraries, "bioactive" compounds in natural products cover a wider area of chemical space [11]. As a valuable resource of active natural products, traditional Chinese medicine (TCM) plays an important role in lung cancer treatment with unique advantages of high efficiency and minimal side effects $[12,13]$. It has an indispensable effect on preventing lung cancer cell proliferation, migration, invasion, enhancing the efficacy, and reducing the side effects of chemotherapy and radiotherapy $[14,15]$.

Wenxia Changfu formula (WCF) is modified from Dahuang Fuzi decoction which was first recorded in Synopsis of Golden Chamber (Jingui Yaolue) written by Zhang Zhongjing from Han Dynasty and is widely used for treating lung cancer in China. It comprises four Chinese herbal ingredients, including Panax ginseng C.A. Mey (Araliaceae) (Renshen), Rheum palmatum L. (Polygonaceae) (Dahuang), Aconitum carmichaelii Debeaux (Ranunculaceae) (Fuzi), and Angelica sinensis (Oliv.) Diels (Apiaceae) (Danggui). Previous studies have demonstrated that WCF has a significant antitumor effect as it inhibits the A549 cell line proliferation and induces lung cancer cell apoptosis [16, 17]. Furthermore, WCF effectively enhances chemotherapeutic efficacy and reverses multidrug resistance mediated by cell adhesion in lung cancer cells, the mechanism of which may be related to the integrin $\beta 1$ signaling pathway $[18,19]$. Herb formula of TCM is characterized by multitarget, multibiological process and multipathway [20]. However, how WCF exerts antitumor effects via promoting apoptosis has not been well illustrated.

In the present study, the effect of WCF on the proliferation of three lung cancer cells was investigated and then the mechanism was further explored by using microarray analysis, high-content short hairpin RNA (shRNA) screening, etc. Finally, we demonstrate that WCF inhibits lung cancer cell proliferation and promotes apoptosis via decreasing PIF1 expression. The result provides insight into the molecular mechanism of WCF in treating lung cancer and helps us better use WCF clinically.

\section{Materials and Methods}

2.1. Preparation of WCF. WCF is composed of Panax ginseng C.A. Mey (Araliaceae) (9g), Aconitum carmichaelii Debeaux (Ranunculaceae) (12 g), Rheum palmatum L. (Polygonaceae) (12 g), and Angelica sinensis (Oliv.) Diels (Apiaceae) $(6 \mathrm{~g})$. All crude herbs were purchased from Shandong ZhongLu Hospital (Jinan, China) in June 2018. The authentication was performed by Dr. Feng Li from Shandong University of Traditional Chinese Medicine (Jinan, China). Voucher specimens (No. WCF4-A, B, C, D) were deposited at Affiliated Hospital of Shandong University of Traditional Chinese Medicine (Jinan, China).

The WCF extract was prepared according to the previously reported methods [19]. In brief, the raw herbs Panax ginseng and Aconitum carmichaelii were decocted for $2 \mathrm{~h}$ after they were macerated for $1 \mathrm{~h}$, and then Angelica sinensis and Rheum palmatum were added and decocted for $0.5 \mathrm{~h}$ and $0.25 \mathrm{~h}$, respectively. Finally, the filtrates were concentrated to $1.6 \mathrm{~g} / \mathrm{mL}$ and stored at $-20^{\circ} \mathrm{C}$. The concentrations of WCF in this study refer to the crude drug concentrations.

2.2. UHPLC/MS Analysis of WCF. The UHPLC/MS analysis was performed according to the procedure we had reported elsewhere [18]. In brief, UHPLC analysis was performed on an UltiMate 3000 RS system (Thermo Fisher Scientific, San Diego, CA). The Thermo Hypersil GOLD column $(2.1 \mathrm{~mm} \times 100 \mathrm{~mm}, 1.9 \mu \mathrm{m})$ was applied for all analyses. The mobile phase was composed of A $(0.1 \%$ formic acid in acetonitrile) and $\mathrm{B}(0.1 \%$ formic acid in water) with a linear gradient elution: $0-5 \mathrm{~min}, \mathrm{~A}: 2-20 \%$; $5-10 \mathrm{~min}, \mathrm{~A}: 20-50 \%$; 10-25 min, A: $50-95 \%$; $26-30 \mathrm{~min}, \mathrm{~A}: 2 \%$. The flow rate of the mobile phase was $0.3 \mathrm{~mL} / \mathrm{min}$, and the injection volume was $5 \mu \mathrm{L}$. The column temperature was maintained at $35^{\circ} \mathrm{C}$.

Mass spectrometry was performed on a $Q$ Exactive highresolution mass spectrometer (Thermo Fisher Scientific, San Diego, CA) and operated using an electrospray source in the positive and negative modes. The operating parameters were as follows: spray voltage, $3.8 \mathrm{kV}$; capillary temperature, $300^{\circ} \mathrm{C}$; sheath gas pressure, $40 \mathrm{arb}$; aux gas heater temperature, $350^{\circ} \mathrm{C}$. Data acquisition and processing were performed using CD 2.1 software (Thermo Fisher), and then they were contrasted with databases (mzCloud, mzVault, and ChemSpider).

2.3. Cell Culture and Reagents. Human NSCLC cell lines A549, H460, and PC-9 and human normal fibroblast cell line MRC-5 were purchased from the Chinese Academy of Sciences (Shanghai, China). A549, H460, and PC-9 cells were cultured in RPMI 1640 (Gibco, USA) media supplemented with 10\% fetal bovine serum (Sijiqing Bioengineering Material Co., Ltd, China), $100 \mathrm{U} / \mathrm{mL}$ penicillin, and $100 \mu \mathrm{g} / \mathrm{mL}$ streptomycin (Gibco, USA) at $37^{\circ} \mathrm{C}$ in a humidified atmosphere containing $5 \% \mathrm{CO}_{2}$. MRC- 5 cells were maintained in DMEM/F12 media supplemented with $10 \%$ fetal bovine serum and $1 \%$ penicillinstreptomycin at $5 \% \mathrm{CO}_{2}, 37^{\circ} \mathrm{C}$.

2.4. 3-(4,5-Dimethylthiazol-2-yl)-2,5-diphenyltetrazolium Bromide (MTT) Assay. Cell proliferation was measured using MTT assay. Cell densities of $5 \times 10^{3}$ (A549, MRC-5, and PC-9) or $4 \times 10^{3}$ (H460) cells per $100 \mu \mathrm{L}$ were seeded into each well in 96-well plates for $12 \mathrm{~h}$; then, they were randomly divided into 5 groups: control group (without WCF) and WCF groups (treated with 100.0, 50.0, 25.0, and $12.5 \mathrm{mg} / \mathrm{mL}$ WCF, respectively) and cultured for $48 \mathrm{~h}$ at $37^{\circ} \mathrm{C}$ in $5 \% \mathrm{CO}_{2}$. Next, $20 \mu \mathrm{L}$ MTT solution was added to each well. After incubating for $4 \mathrm{~h}$, the medium was removed and the formazan crystals were dissolved by adding $150 \mu \mathrm{L}$ DMSO. The absorbance was measured using an ELISA reader (SpectraMax M3, Molecular Devices, USA) at a wavelength of $490 \mathrm{~nm}$.

2.5. Microarray Analysis and Quantitative Real-Time PCR. Total RNA was extracted with TRIzol reagent (Superfec, China) from lung cancer cell line samples and qualified using 
a Thremo NanoDrop 2000 (Thermo Fisher Scientific, USA) and an Agilent 2100 Bioanalyzer (Agilent Technologies, USA). Four WCF-treated samples and four negative control samples were used for microarray analysis using the human GeneChip PrimeView array (Affymetrix). Images were captured using a GeneChip Scanner 3000 and analyzed with GeneChip GCOS 1.4 software (Affymetrix). Differentially expressed genes (DEGs) were identified as the expression fold change was $>2$ and the $P$ value was $<0.05$. For RT-PCR analysis, cDNA synthesis was performed using the M-MLV reverse transcriptase kit (Promega) after total RNA extraction. The real-time PCR was performed in triplicate on a LightCycler 480 System (Roche), and the data were analyzed by using the $2^{-\triangle \triangle \mathrm{Ct}}$ method. GAPDH was used as an internal control.

2.6. High-Content Screening and Cell Growth Curve Analysis. The effects of 21 candidate target genes on A549 cell proliferation were detected by high-content screening (HCS). In brief, A549 cells were transfected with shRNA lentivirus targeting candidate target genes or negative control lentivirus after they were seeded at 2000 cells per well in 96-well plates. The GFP expression was observed by using a fluorescence imaging microscope. Cells were collected for further experiments when they reached $80 \%$ confluence. Growth of cultured cells was monitored every day for continuous 5 days using the Celigo Image Cytometer (Nexcelom). By adjusting the input parameters of the analysis settings, the number of cells with green fluorescence in each scanning well was calculated. Based on these data, cell proliferation curves were established for target genes or negative control. The cell proliferation ratio of each gene was obtained by comparing the number of cells at each time point with the cell count on day 1 , and the cell proliferation curve was produced using the fold change in proliferation. The fold change of each target gene or negative control was calculated as cell count on day $5 /$ cell count on day 1 . The gene with a fold change of $\geq 2$ would be identified as a potential target gene which was related to cell proliferation.

2.7. Apoptosis Analysis. The Apoptosis Detection Kit (eBioscience) was used for apoptosis analysis according to the manufacturer's instructions. Cells were washed with icecold D-Hanks followed by a binding buffer, and then they were resuspended in $1000 \mu \mathrm{L}$ binding buffer containing $10 \mu \mathrm{L}$ Annexin V-APC staining solution. The cells were incubated in the dark for $15 \mathrm{~min}$ at room temperature, and then the percentage of apoptosis was analyzed by FACS (Millipore). All samples were tested three times.

2.8. Caspase 3/7 Activity Analysis. The activity of caspase 3/7 was detected by Caspase-Glo ${ }^{\circledast}$ 3/7 Assay (Promega) according to the kit instruction. Cells were seeded in 96-well plates at $1 \times 10^{4}$ cells per well, and $100 \mu \mathrm{L}$ Caspase-Glo ${ }^{\circledR} 3 / 7$ Reagent was added to each well. The plates were placed on a shaker and shaken at 300-500 rpm for $30 \mathrm{~min}$. Then, the cells were incubated at room temperature for 1-2 $\mathrm{h}$ according to the cell conditions. The luminescence was recorded with the GloMax ${ }^{\circledast}$ System.

2.9. Statistical Analysis. Data are presented as mean \pm SD (standard deviation). Statistical analysis was conducted using SPSS 26.0 (IBM). Statistical differences between two groups were assessed using the independent Student's $t$ test, and differences among three or more groups were assessed using one-way ANOVA. A $P$ value of $<0.05$ was considered statistically significant.

\section{Results}

3.1. UHPLC/MS Analysis of the Chemical Profile of WCF. UHPLC/MS was applied to analyze and identify the chemical composition of WCF. Through the standard comparison, literature review, and disassociation rules, 15 components were identified. The results are shown in Table 1.

3.2. WCF Inhibits Lung Cancer Cell Proliferation. To investigate the effect of WCF on lung cancer cell proliferation, we assessed the cell viability of A549, H460, and PC-9 cells treated with WCF or negative control at $48 \mathrm{~h}$ by MTT assay. The results showed that the viability of these three cells decreased in a dose-dependent way when exposed to WCF for $48 \mathrm{~h}$ (Figure $1(\mathrm{a})-1(\mathrm{c}))$. The $\mathrm{IC}_{50}$ values were $66.29 \mathrm{mg} /$ $\mathrm{mL}$ (A549), $49.53 \mathrm{mg} / \mathrm{mL}$ (H460), and $20.46 \mathrm{mg} / \mathrm{mL}$ (PC-9), respectively. WCF at a concentration of $50.00 \mathrm{mg} / \mathrm{mL}$ (A549 and $\mathrm{H} 460$ ) or $20.00 \mathrm{mg} / \mathrm{mL}$ (PC-9) was selected for subsequent experiments. Besides, there was little effect on the viability of MRC- 5 cells treated with WCF concentrations $\leq 50 \mathrm{mg} / \mathrm{mL}$ (Figure 1(d)).

\subsection{Identification of PIF1 as a Potential Target for WCF in} Treating Lung Cancer. Microarray experiments comparing 4 pairs of WCF-treated samples and negative control samples revealed 3076 DEGs, 1542 of which in WCF-treated samples were upregulated and 1534 were downregulated (Figure 2(a)). To identify novel oncogenes, we focused on 21 downregulated genes that have not been extensively investigated for potential association with lung cancer, including PIF1, GYG2, and PGM2L1 (Figure 2(b)). All 21 candidate genes were silenced in A549 cells in vitro, and the results showed that the silence of PIF1 strongly inhibited cancer cell growth (Figure 2(b) and 2(c)). In addition, PIF1 mRNA expression levels in A549, H460, and PC-9 cells were significantly downregulated after WCF treatment (Figures 2(d)-2(f)), indicating that PIF1 may serve as a potential target for WCF in the regulation of lung cancer cells.

\subsection{PIF1 Promotes Lung Cancer Cell Proliferation In Vitro.}

To further determine PIF1 function in lung cancer cell proliferation, stable PIF1 knockdown in the A549 cell line was established with a lentiviral delivery system, and we confirmed downregulation of both PIF1 protein and mRNA in this cell line (Figure 3(a), 3(b)). PIF1 knockdown 
TABLE 1: Identification of some chemical constituents in WCF.

\begin{tabular}{lcccc}
\hline No. & Name & Formula & RT (min) & mzCloud best match \\
\hline 1 & DL-arginine & $\mathrm{C}_{6} \mathrm{H}_{14} \mathrm{~N}_{4} \mathrm{O}_{2}$ & 1.279 & 93.3 \\
2 & L-histidine & $\mathrm{C}_{6} \mathrm{H}_{9} \mathrm{~N}_{3} \mathrm{O}_{2}$ & 1.296 & 91.4 \\
3 & Trigonelline & $\mathrm{C}_{7} \mathrm{H}_{7} \mathrm{NO}_{2}$ & 1.398 & 94.6 \\
4 & Proline & $\mathrm{C}_{5} \mathrm{H}_{9} \mathrm{NO}_{2}$ & 1.476 & 85.1 \\
5 & Pyridoxine & $\mathrm{C}_{8} \mathrm{H}_{11} \mathrm{NO}_{3}$ & 2.849 & 86.5 \\
6 & $\mathrm{C}_{6} \mathrm{H}_{7} \mathrm{~N}_{5}$ & 4.161 & 89.4 \\
7 & N6-methyladenine & $\mathrm{C}_{10} \mathrm{H}_{9} \mathrm{NO}_{2}$ & 5.915 & 8.1 \\
8 & Indole-3-acetic acid & $\mathrm{C}_{16} \mathrm{H}_{18} \mathrm{O}_{9}$ & 7.099 & 76.2 \\
9 & Neochlorogenic acid & $\mathrm{C}_{11} \mathrm{H}_{13} \mathrm{NO}_{3}$ & 9.022 & 83 \\
10 & N-acetyl-L-phenylalanine & $\mathrm{C}_{16} \mathrm{H}_{12} \mathrm{O}_{5}$ & 13.195 & 80.2 \\
11 & Glycitein & $\mathrm{C}_{30} \mathrm{H}_{50} \mathrm{O}_{2}$ & 14.324 & 82.2 \\
12 & Betulin & $\mathrm{C}_{15} \mathrm{H}_{10} \mathrm{O}_{5}$ & 15.435 & 93 \\
13 & Genistein & $\mathrm{C}_{18} \mathrm{H}_{30} \mathrm{O}_{2}$ & 16.855 & 92.3 \\
14 & $\alpha$-eleostearic acid & $\mathrm{C}_{18} \mathrm{H}_{35} \mathrm{NO}_{15}$ & 17.057 & 89.6 \\
15 & Oleamide & $\mathrm{C}_{15} \mathrm{H}_{10} \mathrm{O}_{4}$ & 17.161 & \\
\hline
\end{tabular}

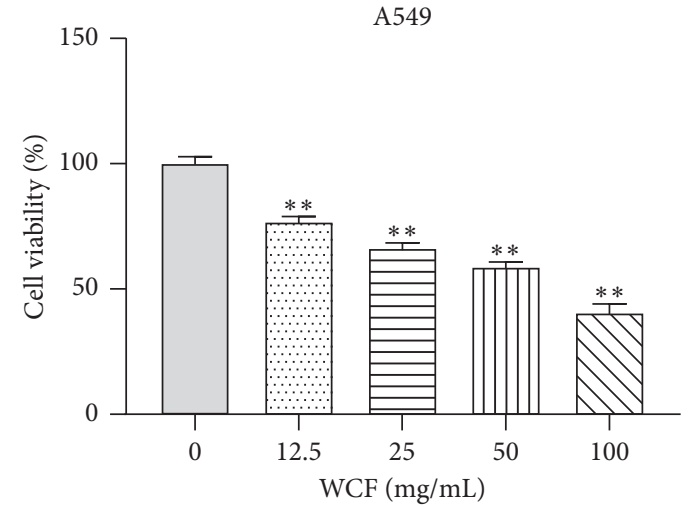

(a)

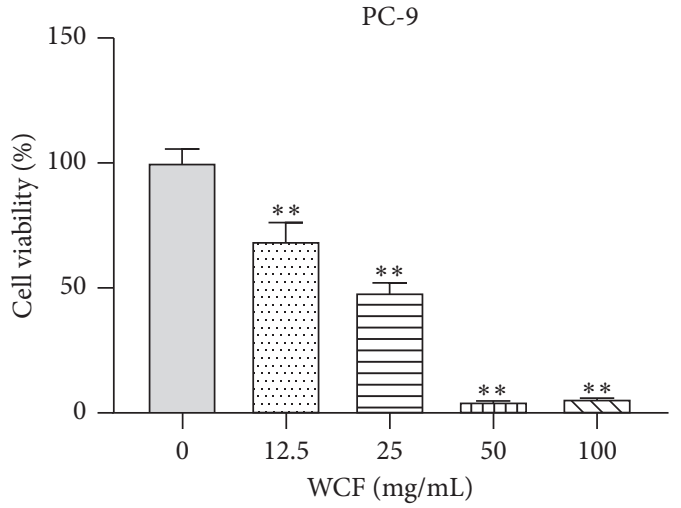

(c)

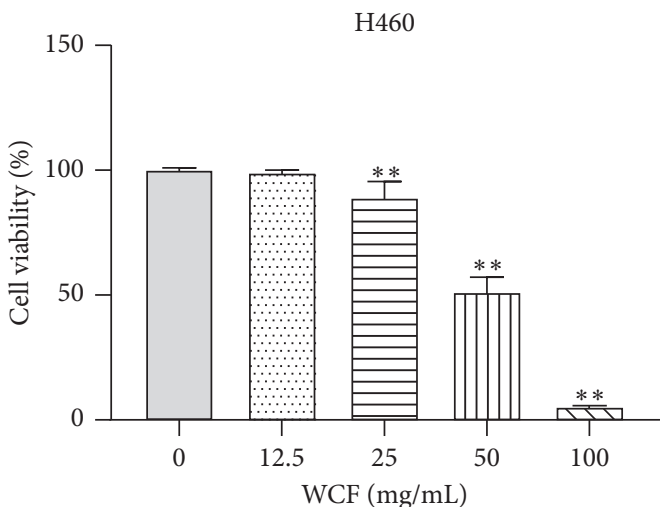

(b)

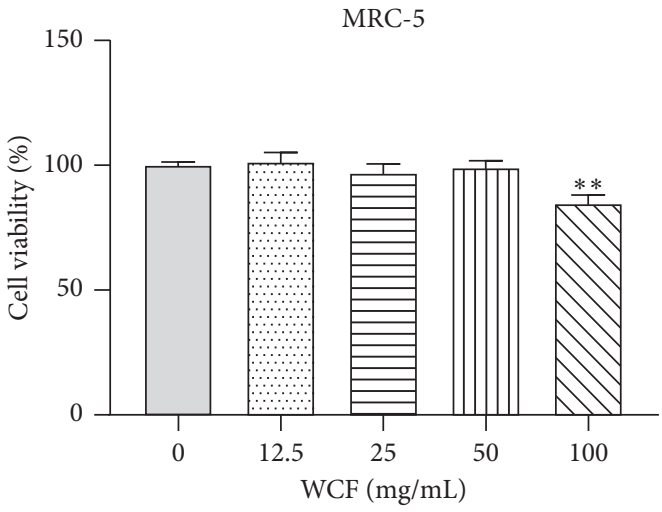

(d)

FIGURE 1: WCF treatment resulted in dose-dependent inhibition of proliferation in NSCLC cell lines. (a) Viability of A549 cells after WCF treatment. (b) Viability of H460 cells after WCF treatment. (c) Viability of PC-9 cells after WCF treatment. (d) Viability of MRC-5 cells after WCF treatment. ${ }^{* *} P<0.01$.

suppressed A549 cell proliferation, as measured by Celigo and cell growth curve analysis using a fluorescence imaging system (Figure 3(c)).

3.5. PIF1 Knockdown Inhibits Lung Cancer Apoptosis. To explore whether PIF1 promotes lung cancer cell proliferation through regulating cell apoptosis, Annexin
V-APC staining was performed by using a flow cytometer. PIF1 knockdown increased the percentage of A549 cells undergoing apoptosis (Figure 4(a), 4(b)), and the activity of caspase $3 / 7$ was increased in the shPIF1 group (Figure $4(\mathrm{c})$ ). These results indicate that PIF1 silencing can inhibit A549 cell proliferation via promoting apoptosis. 


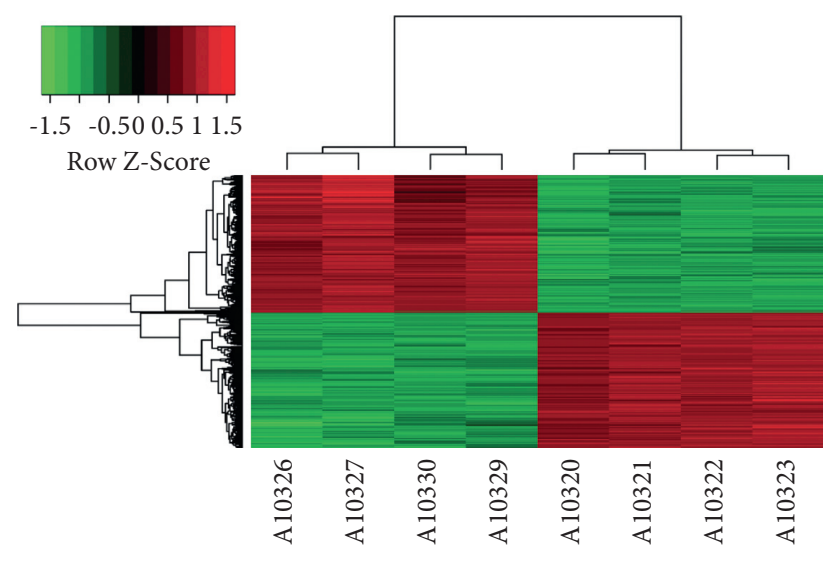

(a)

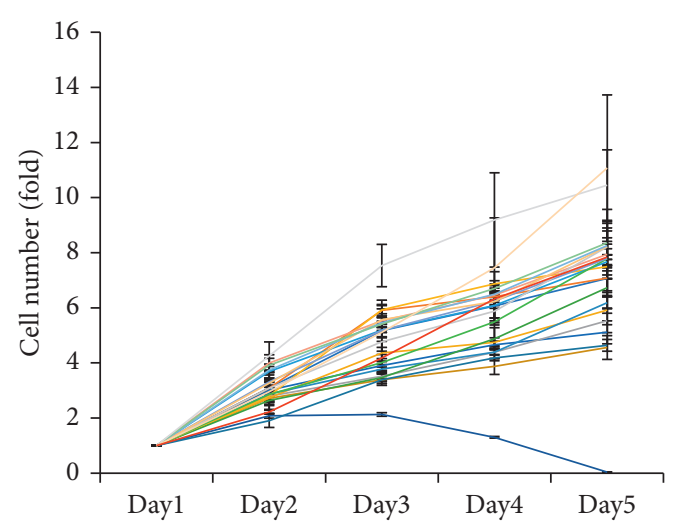

$\begin{array}{ll}\text { - shPIF1 } & - \text { shIFITM2 } \\ \text { shGYG2 } & \text { shIFT81 } \\ \text { shPGM2L1 } & \text { shPDZK1 } \\ \text { shKIFC3 } & \text { shCAMK2N1 } \\ \text { shPBX3 } & \text { shRBMS2 } \\ \text { shCOCH } & \text { shST3GAL5 } \\ \text { shTRNP1 } & \text { shAMIGO2 } \\ \text { shPSD3 } & \text { shSPINK6 } \\ \text { shCNN3 } & \text { shC1RL } \\ \text { shTHSD7A } & \text { shPRSS23 } \\ \text { shFKBP9 } & \text { shCtrl }\end{array}$

(c)

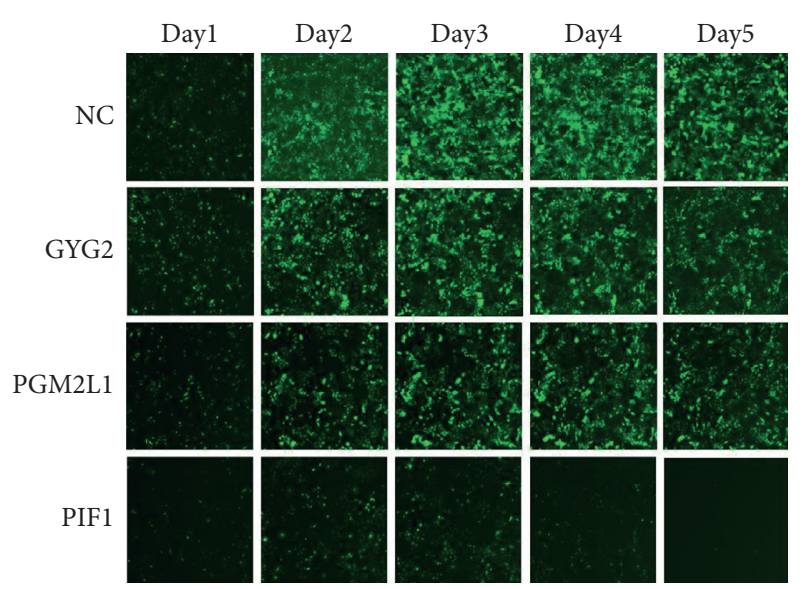

(b)

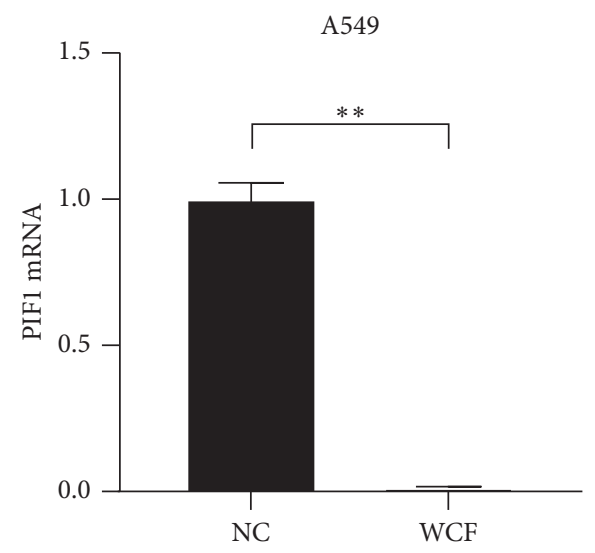

Figure 2: Continued.

(d) 


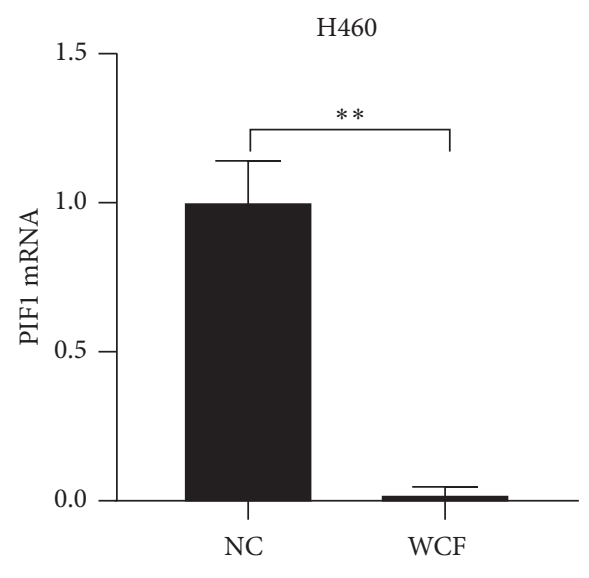

(e)

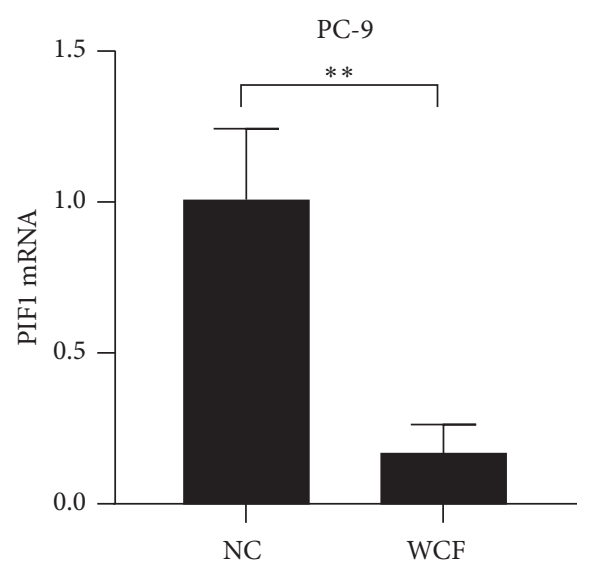

(f)

FIgURE 2: Microarray analysis, quantitative real-time PCR, and high-content screening identified PIF1 as a potential target for WCF in inhibiting lung cancer cell proliferation. (a) Heat map which was used to compare gene expression profiles in A549 cells. (b) Representative fluorescence images of high-content screening for PIF1, GYG2, and PGM2L1 on the growth of A549 cells. (c) A total of 21 genes were selected for validation by high-content screening, and data were presented as fold change and normalized to cell number on day 1 . (d) PIF1 mRNA expression in A549 cells treated with WCF or negative control. (e) PIF1 mRNA expression in H460 cells treated with WCF or negative control. (f) PIF1 mRNA expression in PC-9 cells treated with WCF or negative control. ${ }^{*}{ }^{*} P^{<} 0.01$.

\section{Discussion}

In this study, we determined that WCF could significantly inhibit lung cancer cell proliferation and PIF1 was significantly downregulated after WCF treatment. PIF1 knockdown suppressed cell proliferation and promoted cell apoptosis of A549 cells. These results suggest that PIF1 may play a critical oncogenic role in tumor growth and serve as an attractive therapeutic target after WCF treatment in lung cancer.

TCM is an important part of complementary and alternative medicine and has been increasingly used in the past few decades, which affects the overall survival of lung cancer patients [21]. According to TCM theory, Yang deficiency or Qi deficiency, blood stasis, and stagnation are important pathogenic factors for lung cancer, and WCF can warm Yang, strengthen Qi, resolve blood stasis, and eliminate stagnation [19, 22, 23]. Our previous study showed that combination of WCF with cisplatin could significantly inhibit the A549 cell line proliferation in vitro and reduce the tumor volume in vivo, compared with the cisplatin group, and the apoptosis body was found in tumor cells in the WCF-treated group $[16,17,19]$. WCF has an inhibitory effect on lung cancer cell growth by inducing apoptosis, while the mechanism is still unclear.

Nowadays, microarray bioinformatics has been widely used to identify differentially expressed genes and functional pathways that participate in lung cancer development [24]. There are thousands of genes within the human body, and the expression of these genes varies from tissue to tissue depending on the cell type; thus, the traditional gene-by-gene method is not powerful enough to define gene changes at the genome level in a single experiment [25]. Therefore, in this study, we used microarray technology to screen the differentially expressed genes between the control A549 cells and the WCF-treated A549 cells to identify the potential targets of WCF in inhibiting lung cancer cell proliferation. Finally, 21 oncogenes were identified, and we selected PIF1 to further confirm its biological function in A549 cells based on the highcontent screening results. PIF1 is a multifunctional helicase and plays important roles in mitochondrial and nuclear genome maintenance, telomere length regulation, unwinding of G-quadruplex structures, and DNA synthesis during break-induced replication [26-28]. It is conserved in most eukaryotes and some prokaryotes $[29,30]$. The human genome encodes a single PIF1 gene; splice variant of human PIF1 (hPIF1) localizes to both the nucleus and mitochondria $[31,32]$.

At present, the cellular functions of hPIF1 are still unclear. A few studies have reported that PIF1 was essential for human tumor cell proliferation, including HCT116 cells, HeLa cells, and HEY cells [33-35]. hPIF1 played an important role in DNA replication, especially under oncogenic stress [31]. It had been reported that PIF1 depletion could reduce the survival of neuroblastoma cells by triggering apoptosis, which was dependent on the activity of caspase-3, while nonmalignant cells were not affected by PIF1 depletion [36]. hPIF1 has therefore been proposed as a cancer therapy target [28]. The mechanism may be related to its effect on maintaining mitochondrial stability in response to reactive oxygen species (ROS) [37]. ROS are essential for the initiation, progression, and metastasis of cancer, and altered ROS levels in cancer cells are one of the reasons for recurrence/relapse [38]. By increasing ROS production, cancer cells and drug resistance can be eliminated, which has been demonstrated by a large number of FDA-approved anticancer drugs [38, 39], while PIF1 is involved in reducing the mitochondrial DNA damage caused by ROS [40]. Currently, the role of PIF1 in lung 


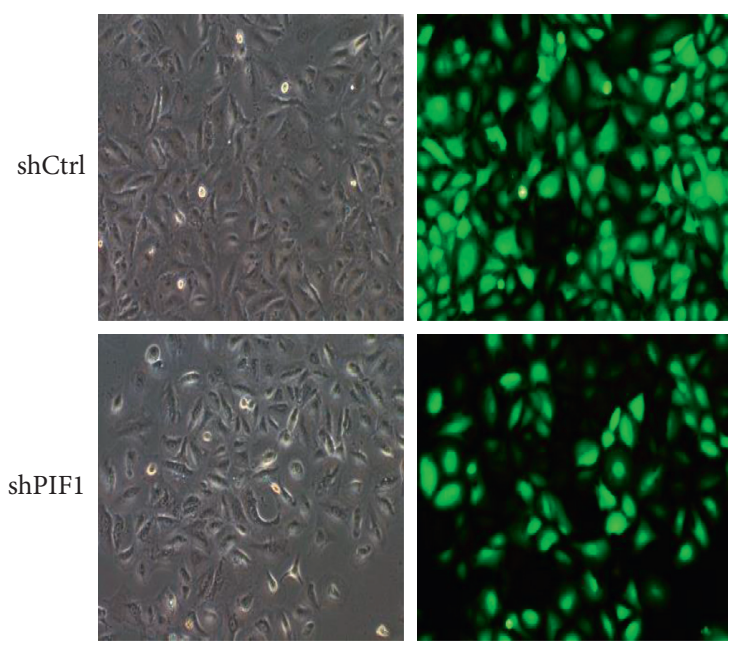

(a)

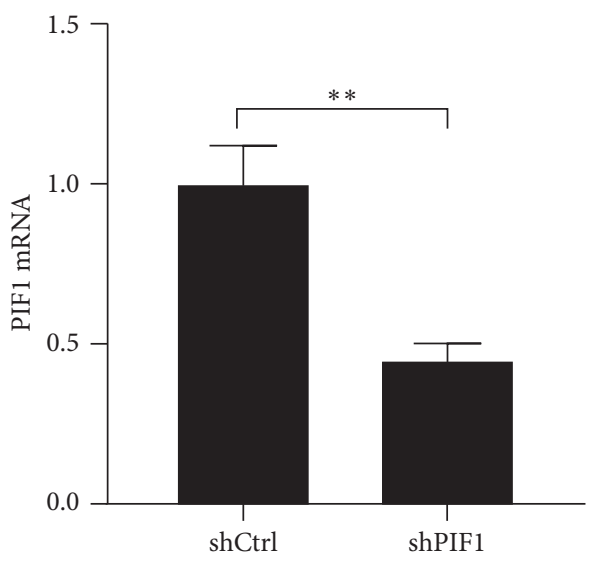

(b)

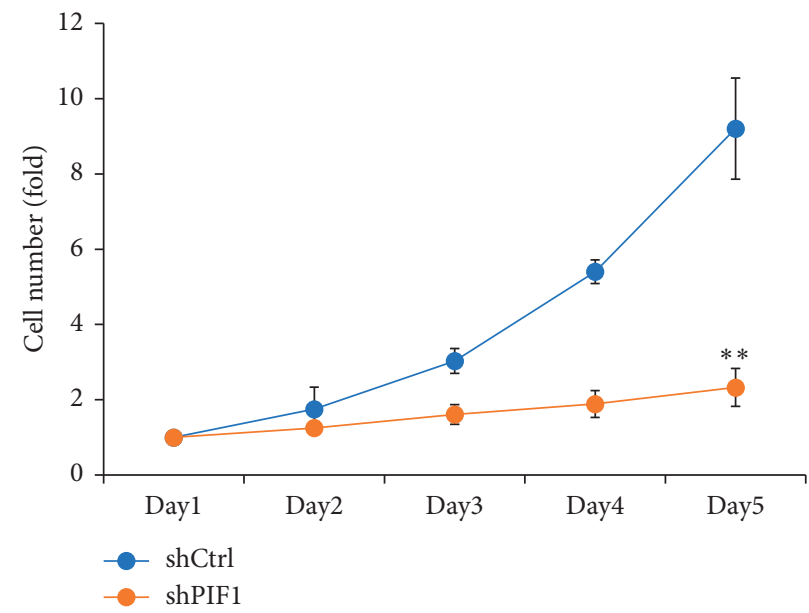

(c)

FIgURE 3: PIF1 promotes A549 cell proliferation in vitro. (a) Representative fluorescence images of A549 cells transfected with shPIF1 or shCtrl for 72 hours. (b) PIF1 mRNA expression in A549 cells transfected with shPIF1 or shCtrl for 72 hours. (c) Cell growth curve analysis comparing PIF1 knockdown (shPIF1) with negative control (shCtrl) A549 cells. ${ }^{*}{ }^{*} P^{<} 0.01$.
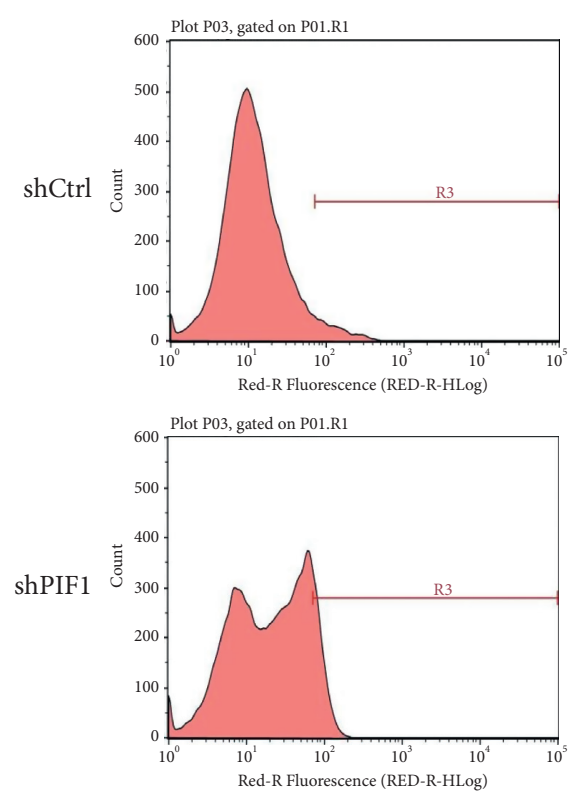
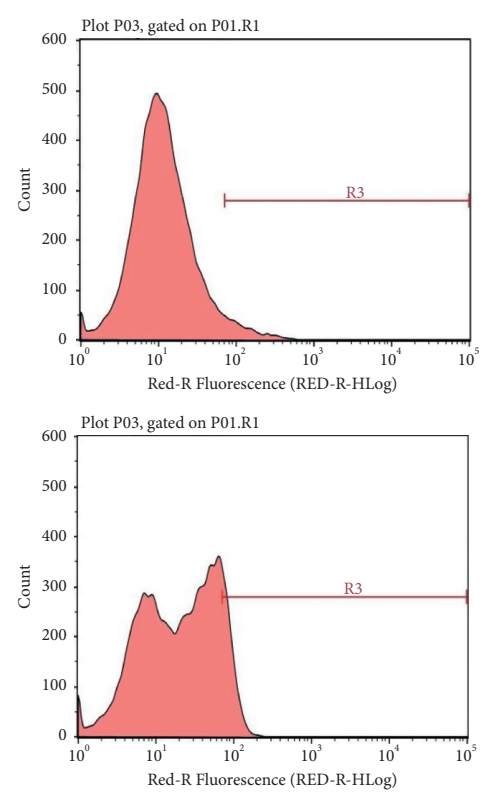

(a)
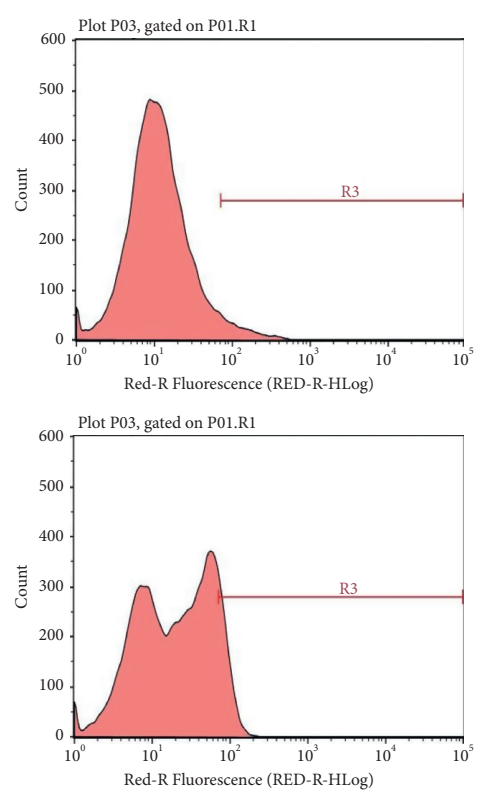

Figure 4: Continued. 


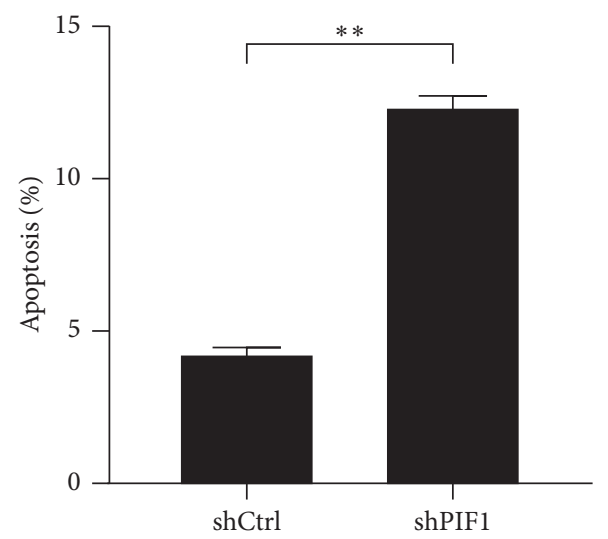

(b)

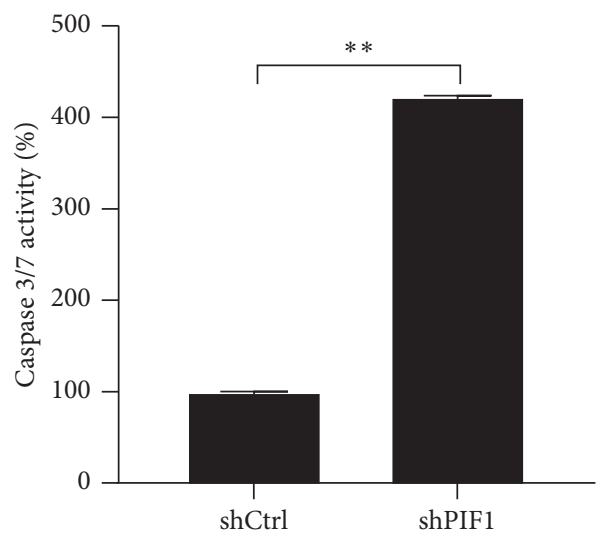

(c)

FIGURE 4: PIF1 regulates A549 cell proliferation via promoting apoptosis. (a, b) Cell apoptosis was analyzed using Annexin V-APC staining, followed by a flow cytometer. (c) Activity of caspase 3/7 was determined in A549 cells transfected with shPIF1 or shCtrl. ${ }^{*} P^{<} 0.01$.

cancer cells is still not reported. In this research, we found that PIF1 played an important role in promoting lung cancer cell proliferation, and it might be a critical target for WCF. However, further studies using appropriate animal models are still needed to confirm the potential of PIF1 as an anticancer drug target.

\section{Conclusions}

We found that WCF inhibited the proliferation of NSCLC cells and downregulated the expression of PIF1 and stable PIF1 knockdown suppressed A549 cell growth and promoted apoptosis. In summary, PIF1 may be a critical gene that regulates lung cancer cell proliferation and apoptosis, which may serve as a potential target of WCF in treating lung cancer.

\section{Data Availability}

The data that support the findings of this study are available from the corresponding author on reasonable request.

\section{Conflicts of Interest}

The authors declare that they have no conflicts of interest.

\section{Authors' Contributions}

XM Ji designed the study, adjusted the experiment, and finalized the paper. DF Kan, JZ Ruan, DL Wang, and XJ Yin performed the experiment and analyzed the data. XJ Yin wrote the manuscript, and XM Ji, JX Wang, Y Chai, SQ Huang, and BY Zhang revised the manuscript. All authors read and approved the final manuscript. Xiangjun Yin, Dongfang Kan, and Jiazhao Ruan contributed equally to this work.

\section{Acknowledgments}

This work was supported by the National Natural Science Foundation of China (Grant nos. 81573871, 81774198, 81703839, and 81273634), the National Key R\&D Program of China (Grant nos. 2019YFC 1708700 and 2019YFC 1708702), the Open Project of Shandong Co-Innovation Center of Classic TCM Formula (Grant no. 2018KFZ03), and Zhejiang Chinese Medical University scientific research fund (Grant nos. 2020ZR11 and 2020ZG14).

\section{References}

[1] K. Park, J. Vansteenkiste, K. H. Lee et al., "Pan-Asian adapted ESMO Clinical Practice Guidelines for the management of patients with locally-advanced unresectable non-small-cell 
lung cancer: a KSMO-ESMO initiative endorsed by CSCO, ISMPO, JSMO, MOS, SSO and TOS," Annals of Oncology, vol. 31, no. 2, pp. 191-201, 2020.

[2] F. Wang, J. Ferlay, I. Soerjomataram, R. L. Siegel, L. A. Torre, and A. Jemal, "Global cancer statistics 2018: GLOBOCAN estimates of incidence and mortality worldwide for 36 cancers in 185 countries," CA: a cancer journal for clinicians, vol. 68, no. 6, pp. 394-424, 2018.

[3] C. Gridelli, A. Rossi, D. P. Carbone et al., "Non-small-cell lung cancer," Nature Reviews Disease Primers, vol. 1, no. 1, p. 15009, 2015.

[4] F. Nasim, B. F. Sabath, and G. A. Eapen, "Lung cancer," Medical Clinics of North America, vol. 103, no. 3, pp. 463-473, 2019.

[5] L. Osmani, F. Askin, E. Gabrielson, and Q. K. Li, "Current WHO guidelines and the critical role of immunohistochemical markers in the subclassification of non-small cell lung carcinoma (NSCLC): moving from targeted therapy to immunotherapy," Seminars in Cancer Biology, vol. 52, no. Pt 1, pp. 103-109, 2018.

[6] A. Vachani, L. V. Sequist, and A. Spira, "AJRCCM: 100YearAnniversary.The shifting landscape for lung cancer: past, present, and future," American Journal of Respiratory and Critical Care Medicine, vol. 195, no. 9, pp. 1150-1160, 2017.

[7] K. D. Miller, L. Nogueira, A. B. Mariotto et al., "Cancer treatment and survivorship statistics, 2019," CA: a cancer journal for clinicians, vol. 69, no. 5, pp. 363-385, 2019.

[8] F. R. Hirsch, G. V. Scagliotti, J. L. Mulshine et al., "Lung cancer: current therapies and new targeted treatments," The Lancet, vol. 389, no. 10066, pp. 299-311, 2017.

[9] A. G. Atanasov, S. B. Zotchev, S. B. Zotchev, V. M. Dirsch, and C. T. Supuran, "Natural products in drug discovery: advances and opportunities," Nature Reviews Drug Discovery, vol. 20, no. 3, pp. 200-216, 2021.

[10] D. J. Newman and G. M. Cragg, "Natural products as sources of new drugs over the nearly four decades from 01/1981 to 09/ 2019," Journal of Natural Products, vol. 83, no. 3, pp. 770-803, 2020.

[11] H. Lachance, S. Wetzel, K. Kumar, and H. Waldmann, "Charting, navigating, and populating natural product chemical space for drug discovery," Journal of Medicinal Chemistry, vol. 55, no. 13, pp. 5989-6001, 2012.

[12] Y. Liu, S. Yang, K. Wang et al., "Cellular senescence and cancer: focusing on traditional Chinese medicine and natural products," Cell Proliferation, vol. 53, no. 10, 2020.

[13] W.-R. Tang, S.-H. Yang, C.-T. Yu et al., "Long-Term effectiveness of combined treatment with traditional Chinese medicine and western medicine on the prognosis of patients with lung cancer," Journal of Alternative \& Complementary Medicine, vol. 22, no. 3, pp. 212-222, 2016.

[14] L. Pang, S. Han, Y. Jiao, S. Jiang, X. He, and P. Li, "Bu Fei Decoction attenuates the tumor associated macrophage stimulated proliferation, migration, invasion and immunosuppression of non-small cell lung cancer, partially via IL-10 and PD-L1 regulation," International Journal of Oncology, vol. 51, no. 1, pp. 25-38, 2017.

[15] Y. Chen, L. Bi, H. Luo et al., "Water extract of ginseng and astragalus regulates macrophage polarization and synergistically enhances DDP's anticancer effect," Journal of Ethnopharmacology, vol. 232, pp. 11-20, 2019.

[16] X.-m. Ji, Z.-c. Wu, G.-w. Liu et al., "Wenxia Changfu Formula (温下肠腑方) induces apoptosis of lung adenocarcinoma in a transplanted tumor model of drug-resistance nude mice,"
Chinese Journal of Integrative Medicine, vol. 22, no. 10, pp. 752-758, 2016.

[17] X.-m. Ji, B. Ouyang, H. Liu et al., "In Vitro and in Vivo inhibitory effect of the combination of Wenxia Changfu Formula (温下肠腑方) with cisplatin in non-small cell lung cancer," Chinese Journal of Integrative Medicine, vol. 17, no. 12, pp. 908-916, 2011.

[18] M.-R. Wang, R.-J. Chen, F. Zhao et al., "Effect of wenxia Changfu formula combined with cisplatin reversing nonsmall cell lung cancer cell adhesion-mediated drug resistance," Frontiers in Pharmacology, vol. 11, p. 500137, 2020.

[19] Y. Zhang, Z. Wu, H. Yu et al., "Chinese herbal medicine wenxia Changfu formula reverses cell adhesion-mediated drug resistance via the integrin $\beta 1$-PI3K-AKT pathway in lung cancer," Journal of Cancer, vol. 10, no. 2, pp. 293-304, 2019.

[20] L. Zeng and K. Yang, "Exploring the pharmacological mechanism of Yanghe Decoction on HER2-positive breast cancer by a network pharmacology approach," Journal of Ethnopharmacology, vol. 199, pp. 68-85, 2017.

[21] Y.-H. Liao, C.-I. Li, C.-C. Lin, J.-G. Lin, J.-H. Chiang, and T.-C. Li, "Traditional Chinese medicine as adjunctive therapy improves the long-term survival of lung cancer patients," Journal of Cancer Research and Clinical Oncology, vol. 143, no. 12, pp. 2425-2435, 2017.

[22] F. Yuan, C. Si-Ning, Z. Ji-Hong et al., "Clinical study of lungsupplementing and stasis-dissolving decoction (Bufei Huayu Tang) combined with gefitnib for treatment of advanced nonsmall cell lung cancer," Pakistan Journal of Pharmaceutical Sciences, vol. 29, no. 6 Spec, pp. 2185-2189, 2016.

[23] Y. Lu, C. Sun, L. Jiao, Y. Liu, Y. Gong, and L. Xu, "Chinese herbal medicine combined with first-generation EGFR-TKIs in treatment of advanced non-small cell lung cancer with EGFR sensitizing mutation: a systematic review and meta-analysis," Frontiers in Pharmacology, vol. 12, Article ID 698371, 2021.

[24] Y.-J. Chen, Y.-N. Guo, K. Shi et al., "Down-regulation of microRNA-144-3p and its clinical value in non-small cell lung cancer: a comprehensive analysis based on microarray, miRNA-sequencing, and quantitative real-time PCR data," Respiratory Research, vol. 20, no. 1, p. 48, 2019.

[25] Z. Tao, A. Shi, R. Li, Y. Wang, X. Wang, and J. Zhao, "Microarray bioinformatics in cancer- a review," Journal of B.U.ON.: Official Journal of the Balkan Union of Oncology, vol. 22, no. 4, pp. 838-843, 2017.

[26] E. Kocak, S. Dykstra, A. Nemeth, C. G. Coughlin, K. Rodgers, and M. McVey, "The Drosophila melanogaster PIF1 helicase promotes survival during replication stress and processive DNA synthesis during double-strand gap repair," Genetics, vol. 213, no. 3, pp. 835-847, 2019.

[27] N. García-Rodríguez, R. P. Wong, and H. D. Ulrich, "The helicase Pif1 functions in the template switching pathway of DNA damage bypass," Nucleic Acids Research, vol. 46, no. 16, pp. 8347-8356, 2018.

[28] S. Dehghani-Tafti, V. Levdikov, A. A. Antson, B. Bax, and C. M. Sanders, "Structural and functional analysis of the nucleotide and DNA binding activities of the human PIF1 helicase," Nucleic Acids Research, vol. 47, no. 6, pp. 3208-3222, 2019.

[29] K. Futami, A. Shimamoto, and Y. Furuichi, "Mitochondrial and nuclear localization of human Pif1 helicase," Biological and Pharmaceutical Bulletin, vol. 30, no. 9, pp. 1685-1692, 2007.

[30] A. K. Byrd and K. D. Raney, "Structure and function of Pif1 helicase," Biochemical Society Transactions, vol. 45, no. 5, pp. 1159-1171, 2017. 
[31] M. E. Gagou, A. Ganesh, G. Phear et al., "Human PIF1 helicase supports DNA replication and cell growth under oncogenic-stress," Oncotarget, vol. 5, no. 22, pp. 11381-11398, 2014.

[32] N. Sabouri, "The functions of the multi-tasking Pfh1Pif1 helicase," Current Genetics, vol. 63, no. 4, pp. 621-626, 2017.

[33] C.-W. Li and B.-S. Chen, "Investigating core genetic-andepigenetic cell cycle networks for stemness and carcinogenic mechanisms, and cancer drug design using big database mining and genome-wide next-generation sequencing data," Cell Cycle, vol. 15, no. 19, pp. 2593-2607, 2016.

[34] M. E. Gagou, A. Ganesh, R. Thompson, G. Phear, C. Sanders, and M. Meuth, "Suppression of apoptosis by PIF1 helicase in human tumor cells," Cancer Research, vol. 71, no. 14, pp. 4998-5008, 2011.

[35] T. Paullin, C. Powell, C. Menzie et al., "Spheroid growth in ovarian cancer alters transcriptome responses for stress pathways and epigenetic responses," PloS One, vol. 12, no. 8, 2017.

[36] B. Chen, Z. Hua, B. Gong et al., "Downregulation of PIF1, a potential new target of MYCN, induces apoptosis and inhibits cell migration in neuroblastoma cells," Life Sciences, vol. 256, p. $117820,2020$.

[37] C. W. Rogers, M. P. Challen, S. Muthumeenakshi, S. Sreenivasaprasad, and J. M. Whipps, "Disruption of the Coniothyrium minitans PIF1 DNA helicase gene impairs growth and capacity for sclerotial mycoparasitism," Microbiology (Reading, England), vol. 154, no. Pt 6, pp. 1628-1636, 2008.

[38] A. Kirtonia, G. Sethi, and M. Garg, "The multifaceted role of reactive oxygen species in tumorigenesis," Cellular and Molecular Life Sciences, vol. 77, no. 22, pp. 4459-4483, 2020.

[39] C. Kim, S. K. Cho, S. Kapoor et al., " $\beta$-caryophyllene oxide inhibits constitutive and inducible STAT3 signaling pathway through induction of the SHP-1 protein tyrosine phosphatase," Molecular Carcinogenesis, vol. 53, no. 10, pp. 793-806, 2014.

[40] T. W. O'Rourke, N. A. Doudican, M. D. Mackereth, P. W. Doetsch, and G. S. Shadel, "Mitochondrial dysfunction due to oxidative mitochondrial DNA damage is reduced through cooperative actions of diverse proteins," Molecular and Cellular Biology, vol. 22, no. 12, pp. 4086-4093, 2002. 